
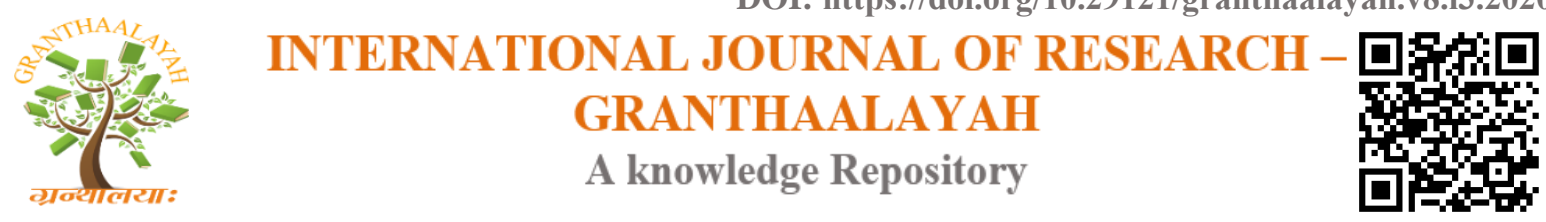

Science

\title{
STUDIES OF THE AVIFAUNA IN URBAN LIMITS OF AJMER, RAJASTHAN, INDIA
}

\author{
Mriganka Upadhyay ${ }^{1,2}$, Reena Vyas ${ }^{1}$, Vivek Sharma ${ }^{3}$, Satya Prakash Mehra ${ }^{4}$ \\ ${ }^{1}$ Department of Zoology, S.P.C. Govt. College Ajmer, (Rajasthan), India \\ ${ }^{2}$ Department of Zoology, Sophia Girls' College Autonomous, Ajmer (Rajasthan), India \\ ${ }^{3}$ M.D.S. University, Ajmer, Rajasthan, India \\ ${ }^{4}$ Rajputana Society of Natural History, Rajasthan, India
}

\begin{abstract}
The urban sprawls are common in the present age. The urban biodiversity conservation is a challenge for the urban planners. The habitats formed within the green and blue spaces are home for the wide variety of floral and faunal diversity. In the series of the conservation actions, the present investigation was undertaken in the urban areas of the central Rajasthan, i.e., Ajmer. It aimed to review and prepare comprehensive database through assessment of the avifaunal species of the municipal area of Ajmer. The seasonal surveys and periodic sampling observations were recorded for twenty-four months from February 2017 to January 2019. The urban area was classified into three regions namely, the Urban Green Patches (UGP), Urban Aquatic Area (UAA) and Human Settlement Area (HSA). The UGA \& HSA harbored 104 species and 41 species respectively whereas the UAA harbored 95 species. The Relative Diversity Index of the various species was calculated. The present investigation recorded 167 species from 58 families. With the earlier studies with a reporting of 235 species from 62 families, there was addition of 13 new species and two families. Thus, the cumulative list of Ajmer District presented an account of 243 bird species from 64 families.
\end{abstract}

Keywords: Urban, Birds, Conservation, Aravalli, Ajmer, Rajasthan.

Cite This Article: Mriganka Upadhyay, Reena Vyas, Vivek Sharma, and Satya Prakash Mehra. (2020). "STUDIES OF THE AVIFAUNA IN URBAN LIMITS OF AJMER, RAJASTHAN, INDIA." International Journal of Research - Granthaalayah, 8(3), 281-296. https://doi.org/10.29121/granthaalayah.v8.i3.2020.159.

\section{Introduction}

The urbanization process has given rise to the human dominated landscapes with complex ecological systems as the urban ecosystems which need to be explored and converted to the opportunity [1-7]. Such man-made ecosystems have certain areas of high biological diversity. These sites are the point of interest for the conservationists for the study of the urban biodiversity especially avifauna. The patterns of biodiversity determine the features of the urban ecosystems. 
Rajasthan (India) owes diversity of the habitats which area also evident in the human settlements. The western low rainfall desert region, central hilly terrains, eastern high rainfall terrains of plains and plateau provide habitats for diverse avifauna. Over 500 avifaunal species are being recorded from the state of Rajasthan. The Aravalli Hills harbor over 300 avifaunal species, near about eighty percent of which are recorded from the central parts $[\mathbf{3 , 5 , 6 , 8}]$.

The study of the birds gives the better understanding of the past and present status of the biodiversity of the area. The environmental challenges could be better understood by the birds [911]. Further, monitoring the species abundance, habitat preference, and correlation between species abundance and habitat provides basic information for determining factors causing population fluctuation of bird species. Richness, abundance and community composition are often used by ecologists to understand the diversity of species in their natural occurrence [12]. The change in vegetation composition could impact the quality and quantity of habitat for birds in terms of food, water and cover which can further affect the diversity, abundance and distribution of birds [13]. In order to prioritize the future conservation of species, understanding the effect of habitat on bird community structure is important [14]. In the long run, the relative value of different habitats and conservation importance of sites can be assessed by investigating the diversity of birds present at those sites [15].

\section{Material and Methods}

\section{Profile of Study Area}

Rajasthan is situated in north - western part of India between the latitude $23^{\circ} 03^{\prime}$ to $30^{\circ} 12^{\prime} \mathrm{N}$ and longitude $69^{0} 30^{\prime}$ to $78^{0} 17^{\prime} \mathrm{E}$. It is the largest state of the Republic of India by area. Geographical features include the Thar Desert, Aravalli Mountain Ranges, Southern Malwa Plateau and Eastern plains. The Ajmer District is located nearly in the center of the Rajasthan and bordered with one of the world oldest mountain ranges i.e. Aravalli Hills (Fig. 1). The Ajmer Municipal area lies in the foothills of the Central Aravalli Hills with the highest peak Taragarh (870 feet) (Fig. 2). It experiences a mean annual rainfall of $573 \mathrm{~mm}$ but scanty and often uncertain. Temperature ranges from $2^{\circ} \mathrm{C}$ to $46^{\circ} \mathrm{C}$. The summers are extremely hot in this part. However, there are many climate changes the Aravalli has witnessed in the recent past, particularly the rainfall, temperature fluctuation and shift of weathers.

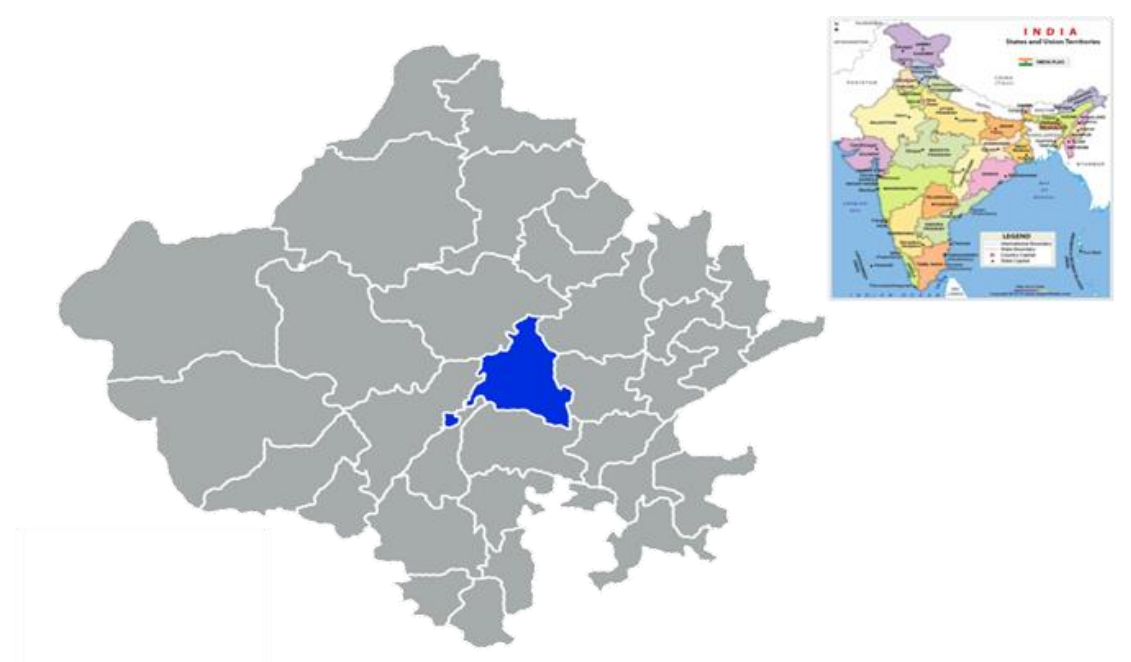

Figure 1: Map of Rajasthan Highlighted with Ajmer District (In Inset Map of India) 


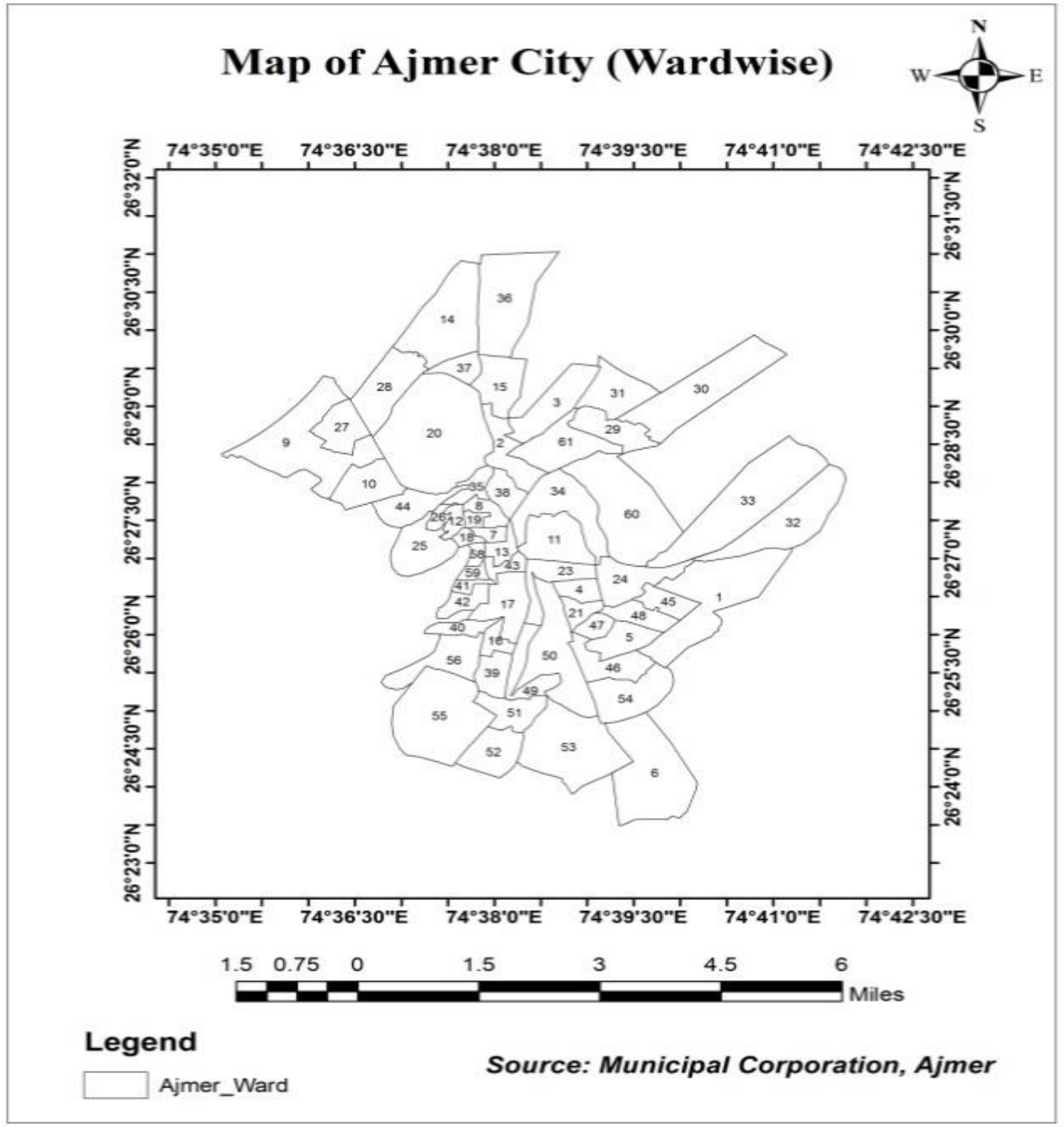

Figure 2: Map of Ajmer Municipal Area of Ajmer District, Rajasthan

For the ease of study, the habitats of the municipal area of Ajmer city in the present study was categorized under two broad groups (see 2, 3).

1) Urban Aquatic Areas (UAA): All perennial and seasonal water bodies within the municipal limits of Ajmer city were categorized under Urban Aquatic Areas (Anasagar Lake, Foy Sagar Lake, Chaurasiywas Talab, Paal Bichla Talab and Khanpura Talab).

2) Urban Terrestrial Areas: The terrestrial area within the municipal limits of Ajmer city which was further classified as:

- Human Settlement Areas (HSA): Areas with direct human involvement and high anthropogenic pressure were included in this category (Constructed areas, buildings, roads etc.).

- Urban Green Patches (UGP): This includes areas with less human intervention and lesser anthropogenic pressure (Institutional and Urban gardens, Agricultural fields, green patches of hillock of Aravalli). 


\section{Field Studies and Surveys}

The field surveys and observations were taken for the period of two years February 2017 to January 2019. Recording of the bird species were also made from the calls. Regular surveys carried out by systematically walking on the fixed routes through the study area. Systematic observations of the species in different habitats of the municipal limits (Fig. 2) were recorded from 6:00 to 9:00 hrs and from 16:00 to 18:00 hrs. General observations were also made during other timings too. The nomenclature is after Manakadan \& Pittie [16], and taxonomic arrangement is following Gill \& Donsker [17]. The status of the species like resident, winter migrant, summer migrant and passage migrant were assigned were assigned as per the observations in each survey based on the presence/absence method along with analysis of the other parameters.

\section{Data Collection and Analysis}

Relative Diversity (RDi): The relative diversity (RDi) of families was calculated using the following formula [18]

$$
\mathbf{R D i}=\frac{\text { Number of bird species in a family }}{\text { Total number of species }} \times 100
$$

Similarity Indices: Similarity indices between the intensive study sites were calculated using Jaccard Index and Sorenson Index [12].

\section{Jaccard Index:}

Jaccard Index:

$$
C_{j}=j /(a+b-j)
$$

Where

$\mathrm{j}=$ the number of species common to both sites

$a=$ the number of species in site $A$ and

$b=$ the number of species in site $b$

\section{Sorenson Index:}

\section{Sorenson Index:}

$$
C_{s}=2 j /(a+b)
$$

Where

$j=$ the number of species common to both sites

$a=$ the number of species in site $A$ and

$b=$ the number of species in site $b$ 


\section{Observation and Results}

Table 1: The Avi-Faunal Composition of Municipal Area of Ajmer District, Rajasthan (Family wise species occurrence at classified microhabitats)

\begin{tabular}{|c|c|c|c|c|c|}
\hline S.No. & Common Name & Scientific Name & UAA & HSA & UGP \\
\hline & Grebes & Podicipedidae & & & \\
\hline \multirow[t]{2}{*}{1} & Little Grebe & Tachybaptus ruficollis (Pallas, 1764) & + & - & - \\
\hline & Pelicans & Pelecanidae & & & \\
\hline 2 & Great White Pelican & $\begin{array}{l}\text { Pelecanus onocrotalus (Linnaeus, } \\
1758 \text { ) }\end{array}$ & + & - & - \\
\hline \multirow[t]{2}{*}{3} & Dalmatian Pelican & Pelecanus crispus (Bruch, 1832) & + & - & - \\
\hline & Cormorants/Shags & Phalacrocoracidae & & & \\
\hline 4 & Little Cormorant & Phalacrocorax niger (Vieillot, 1817) & + & - & - \\
\hline 5 & Indian Shag & $\begin{array}{l}\text { Phalacrocorax fuscicollis (Stephens, } \\
1826 \text { ) }\end{array}$ & + & - & - \\
\hline \multirow[t]{2}{*}{6} & Great Cormorant & Phalacrocorax carbo (Linnaeus, 1758) & + & - & - \\
\hline & Darters & Anhingidae & & & \\
\hline \multirow[t]{2}{*}{7} & Darter & $\begin{array}{l}\text { Anhinga melanogaster (Pennant, } \\
\text { 1769) }\end{array}$ & + & - & - \\
\hline & $\begin{array}{l}\text { Herons, Egrets \& } \\
\text { Bitterns }\end{array}$ & Ardeidae & & & \\
\hline 8 & Little Egret & Egretta garzetta (Linnaeus, 1766) & + & - & - \\
\hline 9 & Grey Heron & Ardea cinerea (Linnaeus, 1758) & + & - & - \\
\hline 10 & Purple Heron & Ardea purpurea (Linnaeus, 1766) & + & - & - \\
\hline 11 & Large Egret & Casmerodius albus (Linnaeus, 1758) & + & - & - \\
\hline 12 & Median Egret & $\begin{array}{l}\text { Mesophoyx intermedia (Wagler, } \\
\text { 1829) }\end{array}$ & + & + & - \\
\hline 13 & Cattle Egret & Bubulcus ibis (Linnaeus, 1758) & + & + & + \\
\hline 14 & Indian Pond-Heron & Ardeola grayii (Sykes, 1832) & + & + & + \\
\hline 15 & Little Green Heron & Butorides striatus (Linnaeus, 1758) & + & - & - \\
\hline \multirow[t]{2}{*}{16} & $\begin{array}{l}\text { Black-crowned Night- } \\
\text { Heron }\end{array}$ & $\begin{array}{l}\text { Nycticorax nycticorax (Linnaeus, } \\
1758 \text { ) }\end{array}$ & + & - & - \\
\hline & Storks & Ciconiidae & & & \\
\hline 17 & Painted Stork & $\begin{array}{l}\text { Mycteria leucocephala (Pennant, } \\
\text { 1769) }\end{array}$ & + & - & + \\
\hline \multirow[t]{2}{*}{18} & Asian Openbill-Stork & Anastomus oscitans (Boddaert, 1783) & + & - & - \\
\hline & Ibises \& Spoonbills & Threskiornithidae & & & \\
\hline 19 & Glossy Ibis & Plegadis falcinellus (Linnaeus, 1766) & + & - & - \\
\hline 20 & Oriental White Ibis & $\begin{array}{l}\text { Threskiornis melanocephalus } \\
\text { (Latham, 1790) }\end{array}$ & + & - & - \\
\hline 21 & Black Ibis & Pseudibis papillosa (Temminck, 1824) & + & - & + \\
\hline \multirow[t]{2}{*}{22} & Eurasian Spoonbill & Platalea leucorodia (Linnaeus, 1758) & + & - & - \\
\hline & Flamingos & Phoenicopteridae & & & \\
\hline 23 & Greater Flamingo & $\begin{array}{l}\text { Phoenicopterus ruber (Linnaeus, } \\
\text { 1758) }\end{array}$ & + & - & - \\
\hline
\end{tabular}


DOI: 10.5281/zenodo.3737844

\begin{tabular}{|c|c|c|c|c|c|}
\hline & $\begin{array}{l}\text { Swans, Geese \& } \\
\text { Ducks }\end{array}$ & Anatidae & & & \\
\hline 24 & Greylag Goose & Anser anser (Linnaeus, 1758) & + & - & - \\
\hline 25 & Bar-headed Goose & Anser indicus (Latham, 1790) & + & - & - \\
\hline 26 & Brahminy Shelduck & Tadorna ferruginea (Pallas, 1764) & + & - & - \\
\hline 27 & Comb Duck & $\begin{array}{l}\text { Sarkidiornis melanotos (Pennant, } \\
1769 \text { ) }\end{array}$ & + & - & - \\
\hline 28 & Gadwall & Anas strepera (Linnaeus, 1758) & + & - & - \\
\hline 29 & Eurasian Wigeon & Anas penelope (Linnaeus, 1758) & + & - & - \\
\hline 30 & Mallard & Anas platyrhynchos (Linnaeus, 1758) & + & - & - \\
\hline 31 & Spot-billed Duck & $\begin{array}{l}\text { Anas poecilorhyncha (J.R. Forester, } \\
\text { 1781) }\end{array}$ & + & - & - \\
\hline 32 & Northern Shoveller & Anas clypeata (Linnaeus, 1758) & + & - & - \\
\hline 33 & Northern Pintail & Anas acuta (Linnaeus, 1758) & + & - & - \\
\hline 34 & Garganey & Anas querquedula (Linnaeus, 1758) & + & - & - \\
\hline 35 & Common Teal & Anas crecca (Linnaeus, 1758) & + & - & - \\
\hline 36 & Red-crested Pochard & Rhodonessa rufina (Pallas, 1773) & + & - & - \\
\hline 37 & Common Pochard & Aythya ferina (Linnaeus, 1758) & + & - & - \\
\hline \multirow[t]{2}{*}{38} & Ferruginous Pochard & Aythya nyroca (Guldenstadt, 1770) & + & - & - \\
\hline & $\begin{array}{l}\text { Hawks, Eagles, } \\
\text { Buzzards, Old World } \\
\text { Vultures, Kites, } \\
\text { Harriers }\end{array}$ & Accipitridae & & & \\
\hline 39 & $\begin{array}{l}\text { Oriental Honey- } \\
\text { Buzzard }\end{array}$ & $\begin{array}{l}\text { Pernis ptilorhynchus (Temminck, } \\
1821 \text { ) }\end{array}$ & - & - & + \\
\hline 40 & Black-shouldered Kite & Elanus caeruleus (Desfontaines, 1789) & - & - & + \\
\hline 41 & Black Kite & Milvus migrans (Boddaert, 1783) & + & + & + \\
\hline 42 & Egyptian Vulture & $\begin{array}{l}\text { Neophron percnopterus (Linnaeus, } \\
1758 \text { ) }\end{array}$ & - & - & + \\
\hline 43 & $\begin{array}{l}\text { Short-toed Snake- } \\
\text { Eagle }\end{array}$ & Circaetus gallicus (Gmelin, 1788) & - & - & + \\
\hline 44 & Western Marsh-Harrier & Circus aeruginosus (Linnaeus, 1758) & + & - & + \\
\hline 45 & Shikra & Accipiter badius (Gmelin, 1788) & - & + & + \\
\hline 46 & Tawny Eagle & Aquila rapax (Temminck, 1828) & - & - & + \\
\hline \multirow[t]{2}{*}{47} & Steppe Eagle & Aquila nipalensis Hodgson, 1833 & - & - & + \\
\hline & Osprey & Pandionidae & & & \\
\hline \multirow[t]{2}{*}{48} & Osprey & Pandion haliaetus (Linnaeus, 1758) & + & - & - \\
\hline & Falcons & Falconidae & & & \\
\hline \multirow[t]{2}{*}{49} & Common Kestrel & Falco tinnunculus (Linnaeus, 1758) & - & - & + \\
\hline & $\begin{array}{l}\text { Pheasants, } \\
\text { Partridges, Quails }\end{array}$ & Phasianidae & & & \\
\hline 50 & Grey Francolin & $\begin{array}{l}\text { Francolinus pondicerianus (Gmelin, } \\
\text { 1789) }\end{array}$ & - & + & + \\
\hline
\end{tabular}


DOI: 10.5281/zenodo.3737844

\begin{tabular}{|c|c|c|c|c|c|}
\hline 51 & Rain Quail & $\begin{array}{l}\text { Coturnix coromandelica (Gmelin, } \\
\text { 1789) }\end{array}$ & - & - & + \\
\hline 52 & Jungle Bush-Quail & Perdicula asiatica (Latham, 1790) & - & - & + \\
\hline \multirow[t]{2}{*}{53} & Indian Peafowl & Pavo cristatus (Linnaeus, 1758) & - & + & + \\
\hline & $\begin{array}{l}\text { Rails, Crakes, } \\
\text { Moorhens, Coots }\end{array}$ & Rallidae & & & \\
\hline 54 & $\begin{array}{l}\text { White-breasted } \\
\text { Waterhen }\end{array}$ & $\begin{array}{l}\text { Amaurornis phoenicurus (Pennant, } \\
1769\end{array}$ & + & - & + \\
\hline 55 & Purple Moorhen & Porphyrio porphyrio (Linnaeus, 1758) & + & - & - \\
\hline 56 & Common Moorhen & Gallinula chloropus (Linnaeus, 1758) & + & - & - \\
\hline \multirow[t]{2}{*}{57} & Common Coot & Fulica atra (Linnaeus, 1758) & + & - & - \\
\hline & Jacanas & Jacanidae & & & \\
\hline 58 & Pheasant-tailed Jacana & $\begin{array}{l}\text { Hydrophasianus chirurgus (Scopoli, } \\
1786 \text { ) }\end{array}$ & + & - & - \\
\hline \multirow[t]{2}{*}{59} & Bronze-winged Jacana & Metopidius indicus (Latham, 1790) & + & - & - \\
\hline & Painted-Snipes & Rostratulidae & & & \\
\hline \multirow[t]{2}{*}{60} & Greater Painted-Snipe & $\begin{array}{l}\text { Rostratula benghalensis (Linnaeus, } \\
\text { 1758) }\end{array}$ & + & - & - \\
\hline & $\begin{array}{l}\text { Plovers, Dotterels, } \\
\text { Lapwings }\end{array}$ & Charadriidae & & & \\
\hline 61 & Little Ringed Plover & Charadrius dubius (Scopoli, 1786) & + & - & - \\
\hline 62 & Kentish Plover & $\begin{array}{l}\text { Charadrius alexandrinus (Linnaeus, } \\
\text { 1758) }\end{array}$ & + & - & - \\
\hline 63 & $\begin{array}{l}\text { Yellow-wattled } \\
\text { Lapwing }\end{array}$ & $\begin{array}{l}\text { Vanellus malabaricus (Boddaert, } \\
\text { 1783) }\end{array}$ & + & - & + \\
\hline \multirow[t]{2}{*}{64} & Red-wattled Lapwing & Vanellus indicus (Boddaert, 1783) & + & + & + \\
\hline & $\begin{array}{l}\text { Sandpipers, Stints, } \\
\text { Snipes, Godwits \& } \\
\text { Curlews }\end{array}$ & Scolopacidae & & & \\
\hline 65 & Common Snipe & Gallinago gallinago (Linnaeus, 1758) & + & - & - \\
\hline 66 & Black-tailed Godwit & Limosa limosa (Linnaeus, 1758) & + & - & - \\
\hline 67 & Spotted Redshank & Tringa erythropus (Pallas, 1764) & + & - & - \\
\hline 68 & Common Redshank & Tringa totanus (Linnaeus, 1758) & + & - & - \\
\hline 69 & Wood Sandpiper & Tringa glareola (Linnaeus, 1758) & + & - & - \\
\hline 70 & Common Sandpiper & Actitis hypoleucos (Linnaeus, 1758) & + & - & + \\
\hline 71 & Little Stint & Calidris minuta (Leisler, 1812) & + & - & + \\
\hline 72 & Temminck's Stint & Calidris temminckii (Leisler, 1812) & + & - & - \\
\hline \multirow[t]{2}{*}{73} & Ruff & Philomachus pugnax (Linnaeus, 1758) & + & - & + \\
\hline & $\begin{array}{l}\text { Ibisbill, Avocets \& } \\
\text { Stilts }\end{array}$ & Recurvirostridae & & & \\
\hline 74 & Black-winged Stilt & $\begin{array}{l}\text { Himantopus himantopus (Linnaeus, } \\
\text { 1758) }\end{array}$ & + & + & + \\
\hline 75 & Pied Avocet & $\begin{array}{l}\text { Recurvirostra avosetta (Linnaeus, } \\
\text { 1758) }\end{array}$ & + & - & - \\
\hline
\end{tabular}


DOI: 10.5281/zenodo.3737844

\begin{tabular}{|c|c|c|c|c|c|}
\hline & $\begin{array}{l}\text { Gulls, Terns \& } \\
\text { Noddies }\end{array}$ & Laridae & & & \\
\hline 76 & Pallas's Gull & Larus ichthyaetus (Pallas, 1773) & + & - & - \\
\hline 77 & Brown-headed Gull & Larus brunnicephalus (Jerdon, 1840) & + & - & - \\
\hline 78 & Black-headed Gull & Larus ridibundus (Linnaeus, 1766) & + & - & - \\
\hline 79 & Gull-billed Tern & Gelochelidon nilotica (Gmelin, 1789) & + & - & - \\
\hline 80 & River Tern & Sterna aurantia (J.E. Gray, 1831) & + & - & - \\
\hline \multirow[t]{2}{*}{81} & Whiskered Tern & Chlidonias hybridus (Pallas, 1811) & + & - & - \\
\hline & Sandgrouse & Pteroclididae & & & \\
\hline 82 & $\begin{array}{l}\text { Chestnut-bellied } \\
\text { Sandgrouse }\end{array}$ & Pterocles exustus (Temminck, 1825) & - & - & + \\
\hline \multirow[t]{2}{*}{83} & Painted Sandgrouse & Pterocles indicus (Gmelin, 1789) & - & - & + \\
\hline & Pigeons \& Doves & Columbidae & & & \\
\hline 84 & Blue Rock Pigeon & Columba livia (Gmelin, 1789) & + & + & + \\
\hline 85 & Little Brown Dove & $\begin{array}{l}\text { Streptopelia senegalensis (Linnaeus, } \\
1766 \text { ) }\end{array}$ & + & + & + \\
\hline 86 & Red Collared-Dove & $\begin{array}{l}\text { Streptopelia tranquebarica (Hermann, } \\
1804 \text { ) }\end{array}$ & - & - & + \\
\hline 87 & $\begin{array}{l}\text { Eurasian Collared- } \\
\text { Dove }\end{array}$ & $\begin{array}{l}\text { Streptopelia decaocto (Frivaldszky, } \\
\text { 1838) }\end{array}$ & + & + & + \\
\hline \multirow[t]{2}{*}{88} & $\begin{array}{l}\text { Yellow-legged Green- } \\
\text { Pigeon }\end{array}$ & Treron phoenicoptera (Latham, 1790) & - & - & + \\
\hline & $\begin{array}{l}\text { Parakeets \& } \\
\text { Hanging-Parrots }\end{array}$ & Psittacidae & & & \\
\hline 89 & Alexandrine Parakeet & Psittacula eupatria (Linnaeus, 1766) & - & - & + \\
\hline 90 & Rose-ringed Parakeet & Psittacula krameri (Scopoli, 1769) & + & + & + \\
\hline \multirow[t]{2}{*}{91} & Plum-headed Parakeet & $\begin{array}{l}\text { Psittacula cyanocephala (Linnaeus, } \\
1766 \text { ) }\end{array}$ & - & - & + \\
\hline & $\begin{array}{l}\text { Cuckoos, Malkohas } \\
\text { \& Coucals }\end{array}$ & Cuculidae & & & \\
\hline 92 & Asian Koel & $\begin{array}{l}\text { Eudynamys scolopacea (Linnaeus, } \\
1758 \text { ) }\end{array}$ & - & + & + \\
\hline \multirow[t]{2}{*}{93} & Greater Coucal & Centropus sinensis (Stephens, 1815) & - & - & + \\
\hline & Owls & Strigidae & & & \\
\hline \multirow[t]{2}{*}{94} & Spotted Owlet & Athene brama (Temminck, 1821) & - & + & + \\
\hline & Nightjars & Caprimulgidae & & & \\
\hline \multirow[t]{2}{*}{95} & $\begin{array}{l}\text { Common Indian } \\
\text { Nightjar }\end{array}$ & Caprimulgus asiaticus (Latham, 1790) & - & - & + \\
\hline & Swifts & Apodidae & & & \\
\hline \multirow[t]{2}{*}{96} & House Swift & Apus affinis (J.E. Gray, 1830) & - & - & + \\
\hline & Kingfishers & Alcedinidae & & & \\
\hline 97 & Small Blue Kingfisher & Alcedo atthis (Linnaeus, 1758) & + & - & - \\
\hline 98 & $\begin{array}{l}\text { White-breasted } \\
\text { Kingfisher }\end{array}$ & Halcyon smyrnensis (Linnaeus, 1758) & + & + & + \\
\hline
\end{tabular}


DOI: 10.5281/zenodo.3737844

\begin{tabular}{|c|c|c|c|c|c|}
\hline 99 & Lesser Pied Kingfisher & Ceryle rudis (Linnaeus, 1758) & + & - & - \\
\hline & Bee-eaters & Meropidae & & & \\
\hline 100 & Small Bee-eater & Merops orientalis (Latham, 1801) & + & + & + \\
\hline 101 & $\begin{array}{l}\text { Blue-cheeked Bee- } \\
\text { eater }\end{array}$ & Merops persicus (Pallas, 1773) & - & - & + \\
\hline \multirow[t]{2}{*}{102} & Blue-tailed Bee-eater & Merops philippinus (Linnaeus, 1766) & - & - & + \\
\hline & Rollers & Coraciidae & & & \\
\hline 103 & European Roller & Coracias garrulus (Linnaeus, 1758) & - & - & + \\
\hline \multirow[t]{2}{*}{104} & Indian Roller & $\begin{array}{l}\text { Coracias benghalensis (Linnaeus, } \\
\text { 1758) }\end{array}$ & + & - & + \\
\hline & Hoopoes & Upupidae & & & \\
\hline \multirow[t]{2}{*}{105} & Common Hoopoe & Upupa epops (Linnaeus, 1758) & - & + & + \\
\hline & Hornbills & Bucerotidae & & & \\
\hline \multirow[t]{2}{*}{106} & Indian Grey Hornbill & Ocyceros birostris (Scopoli, 1786) & - & + & + \\
\hline & Barbets & Capitonidae & & & \\
\hline \multirow[t]{2}{*}{107} & Coppersmith Barbet & $\begin{array}{l}\text { Megalaima haemacephala (P.L.S. } \\
\text { Müller, 1776) }\end{array}$ & - & + & + \\
\hline & Woodpeckers & Picidae & & & \\
\hline 108 & Eurasian Wryneck & Jynx torquilla (Linnaeus, 1758) & - & - & + \\
\hline \multirow[t]{2}{*}{109} & $\begin{array}{l}\text { Lesser Golden-backed } \\
\text { Woodpecker }\end{array}$ & $\begin{array}{l}\text { Dinopium benghalense (Linnaeus, } \\
1758\end{array}$ & - & + & + \\
\hline & Larks & Alaudidae & & & \\
\hline 110 & Singing Bush-Lark & Mirafra cantillans (Blyth, 1845) & - & - & + \\
\hline \multirow[t]{2}{*}{111} & Common Crested Lark & Galerida cristata (Linnaeus, 1758) & - & - & + \\
\hline & Swallows \& Martins & Hirundinidae & & & \\
\hline 112 & Dusky Crag-Martin & Hirundo concolor (Sykes, 1833) & - & + & + \\
\hline 113 & Wire-tailed Swallow & Hirundo smithii (Leach, 1818) & + & + & + \\
\hline 114 & Red-rumped Swallow & Hirundo daurica (Linnaeus, 1771) & - & - & + \\
\hline \multirow[t]{2}{*}{115} & $\begin{array}{l}\text { Streak-throated } \\
\text { Swallow }\end{array}$ & Hirundo fluvicola (Blyth, 1855) & - & - & + \\
\hline & Wagtails \& Pipits & Motacillidae & & & \\
\hline 116 & Large Pied Wagtail & $\begin{array}{l}\text { Motacilla maderaspatensis (Gmelin, } \\
\text { 1789) }\end{array}$ & + & - & - \\
\hline 117 & Citrine Wagtail & Motacilla citreola (Pallas, 1776) & + & - & - \\
\hline 118 & Yellow Wagtail & Motacilla flava (Linnaeus, 1758) & + & - & - \\
\hline \multirow[t]{2}{*}{119} & Paddyfield Pipit & Anthus rufulus (Vieillot, 1818) & + & - & + \\
\hline & $\begin{array}{l}\text { Cuckoo-Shrikes, } \\
\text { Flycatcher-Shrikes, } \\
\text { Trillers, Minivets, } \\
\text { Woodshrikes }\end{array}$ & Campephagidae & & & \\
\hline 120 & Small Minivet & $\begin{array}{l}\text { Pericrocotus cinnamomeus (Linnaeus, } \\
1766 \text { ) }\end{array}$ & - & - & + \\
\hline 121 & Common Woodshrike & $\begin{array}{l}\text { Tephrodornis pondicerianus (Gmelin, } \\
1789 \text { ) }\end{array}$ & - & + & + \\
\hline
\end{tabular}


DOI: 10.5281/zenodo.3737844

\begin{tabular}{|c|c|c|c|c|c|}
\hline & Bulbuls \& Finchbills & Pycnonotidae & & & \\
\hline 122 & White-eared Bulbul & Pycnonotus leucotis (Gould, 1836) & - & - & + \\
\hline \multirow[t]{2}{*}{123} & Red-vented Bulbul & Pycnonotus cafer (Linnaeus, 1766) & + & + & + \\
\hline & Shrikes & Laniidae & & & \\
\hline 124 & Bay-backed Shrike & Lanius vittatus (Valenciennes, 1826) & - & - & + \\
\hline 125 & Rufous-backed Shrike & Lanius schach (Linnaeus, 1758) & - & - & + \\
\hline \multirow[t]{2}{*}{126} & Southern Grey Shrike & $\begin{array}{l}\text { Lanius meridionalis (Temminck, } \\
1820 \text { ) }\end{array}$ & - & + & + \\
\hline & $\begin{array}{l}\text { Thrushes, } \\
\text { Shortwings, Robins, } \\
\text { Forktails, Wheaters }\end{array}$ & Turdinae & & & \\
\hline 127 & Bluethroat & Luscinia svecica (Linnaeus, 1758) & + & - & - \\
\hline 128 & Oriental Magpie-Robin & Copsychus saularis (Linnaeus, 1758) & - & - & + \\
\hline 129 & Indian Robin & Saxicoloides fulicata (Linnaeus, 1776) & - & + & + \\
\hline 130 & Black Redstart & Phoenicurus ochruros (Gmelin, 1774) & - & - & + \\
\hline 131 & Common Stonechat & Saxicola torquata (Linnaeus, 1766) & - & - & + \\
\hline 132 & Pied Bushchat & Saxicola caprata (Linnaeus, 1766) & - & - & + \\
\hline 133 & Desert Wheatear & Oenanthe deserti (Temminck, 1825) & - & - & + \\
\hline 134 & Isabelline Wheatear & $\begin{array}{l}\text { Oenanthe isabellina (Temminck, } \\
\text { 1829) }\end{array}$ & - & - & + \\
\hline \multirow[t]{2}{*}{135} & Indian Chat & Cercomela fusca (Blyth, 1851) & - & + & + \\
\hline & $\begin{array}{l}\text { Babblers, } \\
\text { Laughingthrushes, } \\
\text { Babaxes, Barwings, } \\
\text { Yuhinas }\end{array}$ & Timaliinae & & & \\
\hline 136 & Yellow-eyed Babbler & Chrysomma sinense (Gmelin, 1789) & + & - & - \\
\hline 137 & Common Babbler & Turdoides caudatus (Dumont, 1823) & - & - & + \\
\hline 138 & Large Grey Babbler & Turdoides malcolmi (Sykes, 1832) & - & + & + \\
\hline \multirow[t]{2}{*}{139} & Jungle Babbler & Turdoides striatus (Dumont, 1823) & - & - & + \\
\hline & $\begin{array}{l}\text { Goldcrest, Prinias, } \\
\text { Tesias, Warblers }\end{array}$ & Sylviinae & & & \\
\hline 140 & Ashy Prinia & Prinia socialis (Sykes, 1832) & - & + & + \\
\hline 141 & Rufous-fronted Prinia & Prinia buchanani (Blyth, 1844) & - & - & + \\
\hline 142 & Plain Prinia & Prinia inornata (Sykes, 1832) & - & - & + \\
\hline 143 & Common Chiffchaff & Phylloscopus collybita (Vieillot, 1817) & - & + & + \\
\hline \multirow[t]{2}{*}{144} & $\begin{array}{l}\text { Common Lesser } \\
\text { Whitethroat }\end{array}$ & Sylvia curruca (Linnaeus, 1758) & - & + & + \\
\hline & Flycatchers & Muscicapinae & & & \\
\hline 145 & $\begin{array}{l}\text { Red-throated } \\
\text { Flycatcher }\end{array}$ & Ficedula parva (Bechstein, 1792) & - & - & + \\
\hline \multirow[t]{2}{*}{146} & $\begin{array}{l}\text { Grey-headed } \\
\text { Flycatcher }\end{array}$ & $\begin{array}{l}\text { Culicicapa ceylonensis (Swainson, } \\
1820 \text { ) }\end{array}$ & - & - & + \\
\hline & $\begin{array}{l}\text { Monarch-Flycatchers } \\
\text { \& Paradise- } \\
\text { Flycatchers }\end{array}$ & Monarchinae & & & \\
\hline
\end{tabular}


DOI: 10.5281/zenodo.3737844

\begin{tabular}{|c|c|c|c|c|c|}
\hline 147 & $\begin{array}{l}\text { Asian Paradise- } \\
\text { Flycatcher }\end{array}$ & Terpsiphone paradisi (Linnaeus, 1758) & - & - & + \\
\hline & Fantail-Flycatchers & Rhipidurinae & & & \\
\hline \multirow[t]{2}{*}{148} & $\begin{array}{l}\text { White-browed Fantail- } \\
\text { Flycatcher }\end{array}$ & Rhipidura aureola (Lesson, 1830) & - & - & + \\
\hline & Tits & Paridae & & & \\
\hline \multirow[t]{2}{*}{149} & Great Tit & Parus major (Linnaeus, 1758) & - & - & + \\
\hline & $\begin{array}{l}\text { Sunbirds \& } \\
\text { Spiderhunters }\end{array}$ & Nectariniidae & & & \\
\hline \multirow[t]{2}{*}{150} & Purple Sunbird & Nectarinia asiatica (Latham, 1790) & - & + & + \\
\hline & White-eyes & Zosteropidae & & & \\
\hline \multirow[t]{2}{*}{151} & Oriental White-eye & $\begin{array}{l}\text { Zosterops palpebrosus (Temminck, } \\
\text { 1824) }\end{array}$ & - & - & + \\
\hline & Buntings & Emberizinae & & & \\
\hline \multirow[t]{2}{*}{152} & Crested Bunting & Melophus lathami (Gray, 1831) & - & - & + \\
\hline & Finches & Fringillidae & & & \\
\hline \multirow[t]{2}{*}{153} & Common Rosefinch & Carpodacus erythrinus (Pallas, 1770) & + & - & + \\
\hline & $\begin{array}{l}\text { Munias (Estrildid } \\
\text { Finches) }\end{array}$ & Estrildidae & & & \\
\hline 154 & White-throated Munia & Lonchura malabarica (Linnaeus, 1758) & - & - & + \\
\hline \multirow[t]{2}{*}{155} & Spotted Munia & Lonchura punctulata (Linnaeus, 1758) & - & - & + \\
\hline & $\begin{array}{l}\text { Sparrows \& } \\
\text { Snowfinches }\end{array}$ & Passerinae & & & \\
\hline 156 & House Sparrow & Passer domesticus (Linnaeus, 1758) & + & + & + \\
\hline \multirow[t]{2}{*}{157} & $\begin{array}{l}\text { Yellow-throated } \\
\text { Sparrow }\end{array}$ & Petronia xanthocollis (Burton, 1838) & - & - & + \\
\hline & Weavers & Ploceinae & & & \\
\hline \multirow[t]{2}{*}{158} & Baya Weaver & Ploceus philippinus (Linnaeus, 1766) & + & - & + \\
\hline & Starlings \& Mynas & Sturnidae & & & \\
\hline 159 & Brahminy Starling & Sturnus pagodarum (Gmelin, 1789) & - & + & + \\
\hline 160 & Rosy Starling & Sturnus roseus (Linnaeus, 1758) & + & - & + \\
\hline 161 & Asian Pied Starling & Sturnus contra (Linnaeus, 1758) & + & + & + \\
\hline 162 & Common Myna & Acridotheres tristis (Linnaeus, 1766) & + & + & + \\
\hline \multirow[t]{2}{*}{163} & Bank Myna & $\begin{array}{l}\text { Acridotheres ginginianus (Latham, } \\
\text { 1790) }\end{array}$ & + & + & + \\
\hline & Orioles & Oriolidae & & & \\
\hline \multirow[t]{2}{*}{164} & $\begin{array}{l}\text { Eurasian Golden } \\
\text { Oriole }\end{array}$ & Oriolus oriolus (Linnaeus, 1758) & - & - & + \\
\hline & Drongos & Dicruridae & & & \\
\hline \multirow[t]{2}{*}{165} & Black Drongo & Dicrurus macrocercus (Vieillot, 1817) & + & + & + \\
\hline & $\begin{array}{l}\text { Crows, Jays, } \\
\text { Treepies, Magpies }\end{array}$ & Corvidae & & & \\
\hline 166 & Indian Treepie & $\begin{array}{l}\text { Dendrocitta vagabunda (Latham, } \\
\text { 1790) }\end{array}$ & - & + & + \\
\hline 167 & House Crow & Corvus splendens (Vieillot, 1817) & + & + & + \\
\hline
\end{tabular}


Table legends

UAA: Urban Aquatic Areas; HSA: Human Settlement Areas; UGP: Urban Green Patches

$(+)$ represents presence of species and (-) represents absence of species

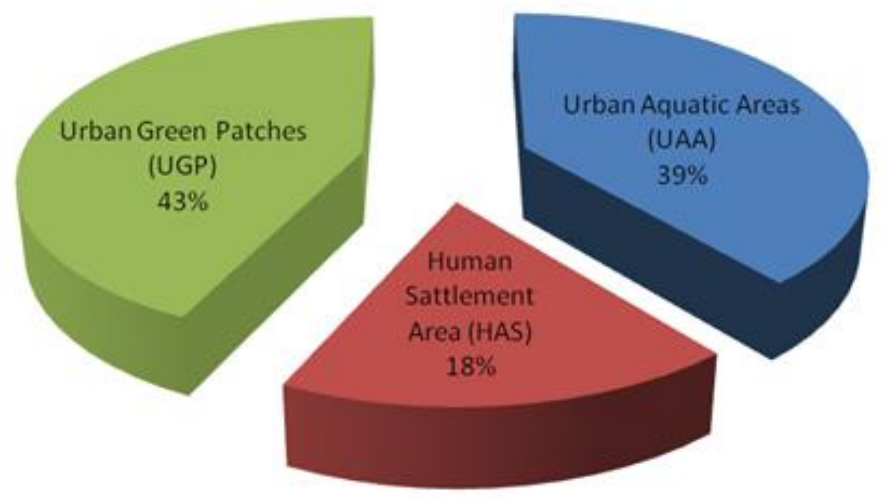

Figure 3: Observed Species at different Microhabitats of Municipal Area of Ajmer District, Rajasthan

Table 2: Relative diversity (RDi) of avian families at Municipal area of Ajmer District, Rajasthan and number of species at various sites

\begin{tabular}{|c|c|c|c|c|c|c|}
\hline S. No. & Family Commonly Known as & Family Name & $\begin{array}{c}\text { Overall } \\
\text { Rdi }\end{array}$ & $\begin{array}{c}\text { No of } \\
\text { Species } \\
\text { in UAA }\end{array}$ & $\begin{array}{c}\text { No of } \\
\text { Species } \\
\text { in HSA }\end{array}$ & $\begin{array}{c}\text { No of } \\
\text { Species } \\
\text { in UGP }\end{array}$ \\
\hline 1 & Grebes & Podicipedidae & 0.6 & 1 & 0 & 0 \\
\hline 2 & Pelicans & Pelecanidae & 1.19 & 2 & 0 & 0 \\
\hline 3 & Cormorants/Shags & Phalacrocoracidae & 1.79 & 3 & 0 & 0 \\
\hline 4 & Darters & Anhingidae & 0.6 & 1 & 0 & 0 \\
\hline 5 & Herons, Egrets \& Bitterns & Ardeidae & 5.36 & 9 & 3 & 2 \\
\hline 6 & Storks & Ciconiidae & 1.19 & 2 & 0 & 1 \\
\hline 7 & Ibises \& Spoonbills & Threskiornithidae & 2.38 & 4 & 0 & 0 \\
\hline 8 & Flamingos & Phoenicopteridae & 0.6 & 1 & 0 & 0 \\
\hline 9 & Swans, Geese \& Ducks & Anatidae & 8.93 & 15 & 0 & 0 \\
\hline 10 & $\begin{array}{l}\text { Hawks, Eagles, Buzzards, Old } \\
\text { World Vultures, Kites, Harriers }\end{array}$ & Accipitridae & 5.36 & 2 & 2 & 9 \\
\hline 11 & Osprey & Pandionidae & 0.6 & 1 & 0 & 0 \\
\hline 12 & Falcons & Falconidae & 0.6 & 0 & 0 & 1 \\
\hline 13 & Pheasants, Partridges, Quails & Phasianidae & 2.38 & 0 & 2 & 4 \\
\hline 14 & Rails, Crakes, Moorhens, Coots & Rallidae & 2.38 & 4 & 0 & 1 \\
\hline 15 & Jacanas & Jacanidae & 1.19 & 2 & 0 & 0 \\
\hline 16 & Painted-Snipes & Rostratulidae & 0.6 & 1 & 0 & 0 \\
\hline 17 & $\begin{array}{l}\text { Sandpipers, Stints, Snipes, } \\
\text { Godwits \& Curlews }\end{array}$ & Scolopacidae & 5.36 & 4 & 1 & 2 \\
\hline 18 & Plovers, Dotterels, Lapwings & Charadriidae & 2.38 & 9 & 0 & 3 \\
\hline 19 & Ibisbill, Avocets \& Stilts & Recurvirostridae & 1.19 & 2 & 1 & 1 \\
\hline 20 & Gulls, Terns \& Noddies & Laridae & 3.57 & 6 & 0 & 0 \\
\hline
\end{tabular}


DOI: 10.5281/zenodo.3737844

\begin{tabular}{|c|c|c|c|c|c|c|}
\hline \\
\hline 21 & Sandgrouse & Pteroclididae & 1.19 & 0 & 0 & 2 \\
\hline 22 & Pigeons \& Doves & Columbidae & 2.98 & 3 & 3 & 5 \\
\hline 23 & Parakeets \& Hanging-Parrots & Psittacidae & 1.79 & 1 & 1 & 3 \\
\hline 24 & Cuckoos, Malkohas \& Coucals & Cuculidae & 1.19 & 0 & 1 & 2 \\
\hline 25 & Owls & Strigidae & 0.6 & 0 & 1 & 1 \\
\hline 26 & Nightjars & Caprimulgidae & 0.6 & 0 & 0 & 1 \\
\hline 27 & Swifts & Apodidae & 0.6 & 0 & 0 & 1 \\
\hline 28 & Kingfishers & Alcedinidae & 1.79 & 3 & 1 & 1 \\
\hline 29 & Bee-eaters & Meropidae & 1.79 & 1 & 1 & 3 \\
\hline 30 & Rollers & Coraciidae & 1.19 & 1 & 0 & 2 \\
\hline 31 & Hoopoes & Upupidae & 0.6 & 0 & 1 & 1 \\
\hline 32 & Hornbills & Bucerotidae & 0.6 & 0 & 1 & 1 \\
\hline 33 & Barbets & Capitonidae & 0.6 & 0 & 1 & 1 \\
\hline 34 & Woodpeckers & Picidae & 1.19 & 0 & 1 & 2 \\
\hline 35 & Larks & Alaudidae & 1.19 & 0 & 0 & 2 \\
\hline 36 & Swallows \& Martins & Hirundinidae & 2.38 & 1 & 2 & 4 \\
\hline 37 & Wagtails \& Pipits & Motacillidae & 2.38 & 4 & 0 & 1 \\
\hline 38 & $\begin{array}{l}\text { Cuckoo-Shrikes, Flycatcher- } \\
\text { Shrikes, Trillers, Minivets, } \\
\text { Woodshrikes }\end{array}$ & Campephagidae & 1.19 & 0 & 1 & 2 \\
\hline 39 & Bulbuls \& Finchbills & Pycnonotidae & 1.19 & 1 & 1 & 2 \\
\hline 40 & Shrikes & Laniidae & 1.79 & 0 & 1 & 3 \\
\hline 41 & $\begin{array}{l}\text { Thrushes, Shortwings, Robins, } \\
\text { Forktails, Wheaters }\end{array}$ & Turdinae & 5.36 & 1 & 2 & 8 \\
\hline 42 & $\begin{array}{l}\text { Babblers, Laughingthrushes, } \\
\text { Babaxes, Barwings, Yuhinas }\end{array}$ & Timaliinae & 2.38 & 1 & 1 & 3 \\
\hline 43 & $\begin{array}{l}\text { Goldcrest, Prinias, Tesias, } \\
\text { Warblers }\end{array}$ & Sylviinae & 2.98 & 0 & 3 & 5 \\
\hline 44 & Flycatchers & Muscicapinae & 1.19 & 0 & 0 & 2 \\
\hline 45 & $\begin{array}{l}\text { Monarch-Flycatchers \& } \\
\text { Paradise-Flycatchers }\end{array}$ & Monarchinae & 0.6 & 0 & 0 & 1 \\
\hline 46 & Fantail-Flycatchers & Rhipidurinae & 0.6 & 0 & 0 & 1 \\
\hline 47 & Tits & Paridae & 0.6 & 0 & 0 & 1 \\
\hline 48 & Sunbirds \& Spiderhunters & Nectariniidae & 0.6 & 0 & 1 & 1 \\
\hline 49 & White-eyes & Zosteropidae & 0.6 & 0 & 0 & 1 \\
\hline 50 & Buntings & Emberizinae & 0.6 & 0 & 0 & 1 \\
\hline 51 & Finches & Fringillidae & 0.6 & 1 & 0 & 1 \\
\hline 52 & Munias (Estrildid Finches) & Estrildidae & 1.19 & 0 & 0 & 2 \\
\hline 53 & Sparrows \& Snowfinches & Passerinae & 1.19 & 1 & 1 & 2 \\
\hline 54 & Weavers & Ploceinae & 0.6 & 1 & 0 & 1 \\
\hline 55 & Starlings \& Mynas & Sturnidae & 3.57 & 4 & 4 & 5 \\
\hline 56 & Orioles & Oriolidae & 0.6 & 0 & 0 & 1 \\
\hline 57 & Drongos & Dicruridae & 0.6 & 1 & 1 & 1 \\
\hline 58 & Crows, Jays, Treepies, Magpies & Corvidae & 1.19 & 1 & 2 & 2 \\
\hline & & & 100 & 95 & 41 & 104 \\
\hline
\end{tabular}


Table 3: Similarity indices (Jaccard Index and Sorenson Index) between the various study sites

\begin{tabular}{|c|c|c|c|c|}
\hline & \multicolumn{4}{|c|}{ Jaccard Index } \\
\hline \multirow{4}{*}{ 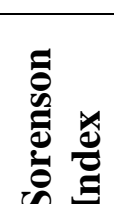 } & & UAA & HAS & UGP \\
\hline & UAA & & 0.17 & \\
\hline & HSA & 0.29 & & 0.3809 \\
\hline & UGP & 0.3300 & 0.5517 & \\
\hline
\end{tabular}

\section{Results and Discussions}

In the investigation period of twenty-four months, a total of 167 species of birds belonging to 58 families were recorded in the study area (Table 1). The three different study sites i.e. Urban Aquatic Area (UAA) had 39\% of the total species observed; Human Settlement Area (HSA) had $18 \%$ and Urban Green Patches (UGP) had $43 \%$ of the total species observed (Fig. 3). The Relative Diversity of different families was calculated to determine the dominance of species occurrence at a particular study site (Table 2). The highest relative diversity was recorded as of Anatidae family with 15 species and 8.93 RDi followed by Ardeidae, Accipitridae, Scolopacidae and Turdinae families with 9 species each and 5.36 RDi respectively representing the dominance of species occurrence belonging to these families at study area (Table 2). The calculation for the similarity indices among different study sites reveled that Urban Green Patches and Human Settlement Areas had more similar habitat structure as the Jaccard index and Sorenson Index values are higher i.e. 0.3809 and 0.5517 respectively. While on the other hand Urban Aquatic Areas and Human Settlement Areas had very less similarity in habitat characteristics hence the values of Jaccard index and Sorenson Index are lower as 0.1709 and 0.2919 respectively (Table 3).

Overall, 95 species belonging to 34 families were present in the Urban Aquatic Area (UAA). The Anatidae family with 15 species dominated the Urban Aquatic Area followed by the Scolopacidae with 9 species. The urban terrestrial area which was further classified for simplification into Human Settlement Area (HAS) and Urban Green Patches (UGP). The Human Settlement Area was represented by 41 species belonging to 27 families. This Area had a lesser number of species and was dominated by Sturnidae family (4 species) followed by Ardeidae, Columbidae, and Sylviinae (3 species each). The Urban Green Patches had 47 families (104 species) and Accipitrae family dominated the area ( 9 species) followed by Turdinae family ( 8 species).

\section{Conclusion}

The present study concluded that the Municipal area of Ajmer district represents near about $68 \%$ of the avifaunal diversity observed in different habitats of the Ajmer District as a whole [2,3,5-8]. The study area includes the foothills of the oldest mountain range the Aravalli that provides an excellent habitat for various floral and faunal components hence the diversity is rich at the study area. The Urban Aquatic Areas (Anasagar Lake, Foy Sagar Lake, Chaurasiywas Talab, Paal Bichla Talab and Khanpura Talab) represented by the 96 species belonging to the 34 families. The Anasagar Lake, which is located in the central part of Ajmer Municipal area supports the 40 species [19], whereas another study conducted after two years by same authors documented 42 species from the same locality [20]. In the continuation another worker documented 48 species of birds from the Anasagar Lake and 42 species from the Foy Sagar Lake [21]. 


\section{References}

[1] Mehra, S. P., Mehra, S., Uddin, M., Verma, V., Sharma, H., Singh, T., Kaur, G., Rimung, T. \& Kumhar, H. R. (2017): Waste as a resource for avifauna: Review and survey of the avifaunal composition in and around waste dumping sites and sewage water collection sites (India). Int $J$ Waste Resour 7(3):289. doi: 10.4172/2252-5211.1000289

[2] Mehra, S. P., Mehra, S. \& Sharma, K. K. (2014): Importance of urban biodiversity - A case study of Udaipur, India. Pp. 403-418. In: Maheshwari, B. L., Purohit, R C., Malano, H. M., Singh, V. P. \& Amerasinghe (eds.) Securing Water, Food, Energy and the Liveability of Cities: Challenges and Opportunities for Peri-urban Futures, Springer Science+Business Media B.V. Dordrecht, The Netherlands. (https://link.springer.com/chapter/10.1007/978-94-017-8878-6_31)

[3] Mehra, S. P., Mehra, S. \& Sen, P. (2013): Urban avifauna of Udaipur and its importance to the local population (Udaipur, Rajasthan, India). International Journal of Biodiversity Watch. Jul-Dec (2013) No. 2: 120-146.

[4] Mehra, S. P. \& Mehra, S. (2013). Short Study to Assess the Potential of Wetlands of Dholpur, Rajasthan, India. Pp 306-313. In: Sheikh, M. M. (ed.) Environmental Consciousness and Human Perceptions, LAP LAMBERT Academic Publishing, Germany.

[5] Mehra, S. (2012): The avifauna of southern Rajasthan with special emphasis on threatened species and bioacoustic applications in their identifications and monitoring. Department of Zoology, M. D. S. University, Ajmer. Ph. D. Thesis.

[6] Mehra, S, Mehra, S. P. \& Sharma, K. K. (2012): Importance of aquatic avifauna in southern Rajasthan, India. Pg. 159-183. In: Rawat., M. \& Dookia, S. (eds.) Biodiversity of Aquatic Resources, Daya Publishing House, Delhi, 2012.

[7] Mehra, S, Mehra, S. P. \& Sharma, K. K. (2011): Aquatic Avifauna: Its Importance for Wetland conservation in Rajasthan, India. Pg. 179-190. In: Mathur, S. M.; Shrivastava, V. K. \& Purohit, R. C. (eds.) Conservation of Lakes and Water Resources Management strategies, Himanshu Publications, Udaipur, 2011.

[8] Mehra, S, Mehra, S. P. \& Sharma, K. K. (2011): Aquatic avifauna of Aravalli Hills Rajasthan, India. Pp. 145-167 (In Gupta, V. K. \& Verma, A. K. (eds.) Animal Diversity, Natural History and Conservation Vol. I, Daya Publishing House, Delhi, 2011.

[9] Mehra, S. P. \& Mehra, S. (2014). Perspective on water and Biodiversity Issues: A Case Study of Keoladeo National Park, Bharatpur, India. Pp. 419-434. In: Maheshwari, B. L., Purohit, R C., Malano, H. M., Singh, V. P. \& Amerasinghe (eds.) Securing Water, Food, Energy and the Liveability of Cities: Challenges and Opportunities for Peri-urban 4Futures, Springer Science+Business Media B.V. Dordrecht, The Netherlands. (https://link.springer.com/chapter/10.1007/978-94-017-8878-6_32)

[10] Taper, M.L., Bohning-Gaese, K. and Brown, J.H. (1995): Individualistic responses of bird species to environmental change. Oecologia 101: 478-486.

[11] Olechnowski, B.F. (2009): An examination of songbird avian diversity, abundance trends, and community composition in two endangered temperate ecosystems: riparian willow habitat of the Greater Yellowstone Ecosystem and a restored tall grass prairie ecosystem, Neal Smith National Wildlife Refuge Iowa State University. Iowa State University.

[12] Magurran, A.E. (1988): Ecological Diversity and its Measurement. Princeton University Press, Princeton, NJ. 192pp

[13] Western, D. and Grimsdell, J.J.R. (1979): Measuring the distribution of animals in relation to the environment. Handbook No. 2, African Wildlife Leadership Foundation, Nairobi

[14] Zakaria, M., Rajpar, M.N. and Sajap, S.A. (2009): Species diversity and feeding guilds of birds in Paya Indah Wetland Reserve, Peninsular Malaysia. Intl. J. Zoological Res. 5(3): 86-100. 
[15] Bensizerara, D., Chenchouni, H., Bachir, A. S. and Houhamdi, M. (2013): Ecological status interactions for assessing bird diversity in relation to a heterogeneous landscape structure. Avian Biology Research 6(1): 67-77.

[16] Manakadan, R. and Pittie, A. (2001): Standardized common and scientific names of the birds of the Indian subcontinent. Buceros 6(1): 1-37.

[17] Gill, F. and Donsker, D. (eds.) (2017): IOC world bird list (v. 7.1). doi: 10.14344/IOC.ML.7.1.

[18] Torre-Cuadros, M.D.L.A.L., Herrando-Perez, S. \& Young, K.R. (2007): Diversity and Structure patterns for tropical montane and premontane forests of central Peru, with an assessment of the use of higher-taxon surrogacy. Biodiversity and Conservation 16:2965-2988.

[19] Yadav, I. and Swarop, R. (2017): Diversity, Abundance and Inter-specific Correlation in Waterbirds at Anasagar Lake, Ajmer. Int. Jon. Sci. and Res. 6(7): 1306-1317.

[20] Swarop, R. and Yadav, I. (2017): Frequency and Status of occurrence of water-birds at Anasagar Lake, Ajmer. Int. J. for Res. Appl. Sc. Engg. Tech. 5(10), 1079-1090.

[21] Dutt, U. and Prakesh, B. (2018): Distribution and Assessment of Water-bird and Water-Associated Birds Diversity from Two Wetlands of Ajmer, Rajasthan. Shrinkhla Ek Shodhparak Vaicharik Patrika 5(12): 82-89.

*Corresponding author.

E-mail address: mrig ankaupadhy ay@gmail.com 